\title{
Teaching Video NeuroImage: Carbamazepine Improves Gait Initiation in Autosomal Recessive Myotonia Congenita
}

Yasuhiro Fuseya, MD, PhD, Nana Ishikawa, MD, Ryogen Sasaki, MD, PhD, and Hirofumi Yamashita, MD, PhD

Neurology ${ }^{\circledR}$ 2022;98:e328. doi:10.1212/WNL.0000000000013010

A 60-year-old man presented with muscle stiffness. He had tended to fall since childhood. His parents were first cousins, and his relatives had no symptoms. Examination showed myotonia in ocular, hand, and limb muscles, followed by improvement with repeated activity (warm-up phenomenon; Video 1). Cold exposure did not aggravate myotonia. His lower legs showed muscle hypertrophy. Genetic tests showed a homozygous p.M560T mutation in the CLCN1 gene, which encodes skeletal muscle chloride channel 1, consistent with autosomal recessive myotonia congenita (Becker disease). ${ }^{1}$ Although treatment with mexiletine or phenytoin did not improve his symptoms, carbamazepine $(350 \mathrm{mg} / \mathrm{d})$ did, especially the walking disturbance (Video 2). Although the warm-up phenomenon is sometimes also observed in sodium channel myotonia, ${ }^{2}$ it is characteristic of myotonia congenita and an important clue for the diagnosis.

\section{Study Funding}

The authors report no targeted funding.

\section{Disclosure}

The authors report no disclosures relevant to the manuscript. Go to Neurology.org/N for full disclosures.

Appendix Authors

\begin{tabular}{lll}
\hline Name & Location & Contribution \\
\hline $\begin{array}{l}\text { Yasuhiro Fuseya, } \\
\text { MD, PhD }\end{array}$ & $\begin{array}{l}\text { Department of Molecular and Cellular Physiology, Kyoto } \\
\text { University, Kyoto, Japan }\end{array}$ & $\begin{array}{l}\text { Managed the patient and drafted } \\
\text { the manuscript and video }\end{array}$ \\
\hline $\begin{array}{l}\text { Nana Ishikawa, } \\
\text { MD }\end{array}$ & Akahoshi Neurology Clinic, Osaka, Japan & Managed the patient \\
\hline $\begin{array}{l}\text { Ryogen Sasaki } \\
\text { MD, PhD }\end{array}$ & $\begin{array}{l}\text { Department of Neurology, Kuwana City Medical Center, } \\
\text { Mie, Japan }\end{array}$ & Performed a genetic test \\
\hline $\begin{array}{l}\text { Hirofumi } \\
\text { Yamashita, MD, } \\
\text { PhD }\end{array}$ & $\begin{array}{l}\text { Department of Neurology, Japanese Red Cross } \\
\text { Wakayama Medical Center, Wakayama, Japan }\end{array}$ & Managed the patient and drafted \\
\end{tabular}

\section{References}

1. Zhang J, George AL, Jr., Griggs RC, et al. Mutations in the human skeletal muscle chloride channel gene (CLCN1) associated with dominant and recessive myotonia congenita. Neurology. 1996;47:993-998.

2. Trip J, Drost G, Ginjaar HB, et al. Redefining the clinical phenotypes of non-dystrophic myotonic syndromes. J Neurol Neurosurg Psychiatry. 2009;80:647-652.
Correspondence

Dr. Yamashita

hirofumi@kuhp.kyoto-u.ac.jp

\section{MORE ONLINE}

\section{Teaching slides}

links.lww.com/WNL/

B639

Videos 


\section{Neurology}

\section{Teaching Video NeuroImage: Carbamazepine Improves Gait Initiation in Autosomal Recessive Myotonia Congenita}

Yasuhiro Fuseya, Nana Ishikawa, Ryogen Sasaki, et al.

Neurology 2022;98;e328 Published Online before print October 21, 2021

DOI 10.1212/WNL.0000000000013010

This information is current as of October 21, 2021

\section{Updated Information \&} Services

References

Subspecialty Collections

Permissions \& Licensing

Reprints including high resolution figures, can be found at: http://n.neurology.org/content/98/3/e328.full

This article cites 2 articles, 2 of which you can access for free at: http://n.neurology.org/content/98/3/e328.full\#ref-list-1

This article, along with others on similar topics, appears in the following collection(s):

All Education

http://n.neurology.org/cgi/collection/all_education

All Movement Disorders

http://n.neurology.org/cgi/collection/all_movement_disorders

Clinical neurology examination

http://n.neurology.org/cgi/collection/clinical_neurology_examination

Ion channel gene defects

http://n.neurology.org/cgi/collection/ion_channel_gene_defects

Muscle disease

http://n.neurology.org/cgi/collection/muscle_disease

Information about reproducing this article in parts (figures,tables) or in its entirety can be found online at:

http://www.neurology.org/about/about_the_journal\#permissions

Information about ordering reprints can be found online:

http://n.neurology.org/subscribers/advertise

Neurology ${ }^{\circledR}$ is the official journal of the American Academy of Neurology. Published continuously since 1951, it is now a weekly with 48 issues per year. Copyright (C 2021 American Academy of Neurology. All rights reserved. Print ISSN: 0028-3878. Online ISSN: 1526-632X.

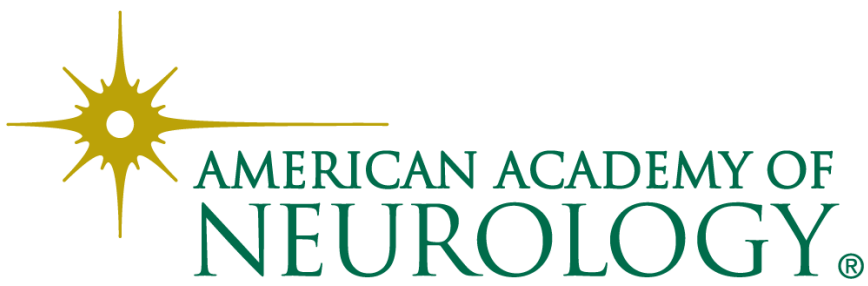

